\title{
SPECT/CT and pulmonary embolism
}

\author{
Jann Mortensen • Henrik Gutte
}

Received: 9 October 2013 / Accepted: 10 October 2013 /Published online: 9 November 2013

(C) The Author(s) 2013. This article is published with open access at Springerlink.com

\begin{abstract}
Acute pulmonary embolism (PE) is diagnosed either by ventilation/perfusion (V/P) scintigraphy or pulmonary CT angiography (CTPA). In recent years both techniques have improved. Many nuclear medicine centres have adopted the single photon emission CT (SPECT) technique as opposed to the planar technique for diagnosing PE. SPECT has been shown to have fewer indeterminate results and a higher diagnostic value. The latest improvement is the combination of a low-dose CT scan with a V/P SPECT scan in a hybrid tomograph. In a study comparing CTPA, planar scintigraphy and SPECT alone, SPECT/CT had the best diagnostic accuracy for PE. In addition, recent developments in the CTPA technique have made it possible to image the pulmonary arteries of the lungs in one breath-hold. This development is based on the change from a single-detector to multidetector $\mathrm{CT}$ technology with an increase in volume coverage per rotation and faster rotation. Furthermore, the dual energy CT technique is a promising modality that can provide functional imaging in combination with anatomical information. Newer high-end CT scanners and SPECT systems are able to visualize smaller subsegmental emboli. However, consensus is lacking regarding the clinical impact and treatment. In the present review, SPECT and SPECT in combination with low-dose CT,
\end{abstract}

J. Mortensen $(\bowtie) \cdot$ H. Gutte

Department of Clinical Physiology, Nuclear Medicine \& PET,

Rigshospitalet, Copenhagen University Hospital,

2100 Copenhagen, Denmark

e-mail: jann.mortensen@regionh.dk

J. Mortensen

Department of Medicine, The Faroese National Hospital, Torshavn, Faroe Islands

H. Gutte

Department of Radiology, Herlev Hospital, Copenhagen University

Hospital, Copenhagen, Denmark

H. Gutte

Cluster for Molecular Imaging, Faculty of Health Sciences,

University of Copenhagen, Copenhagen, Denmark
CTPA and dual energy CT are discussed in the context of diagnosing PE.

Keywords SPECT $\cdot$ SPECT/CT $\cdot$ Pulmonary CT angiography $\cdot$ Lung scintigraphy $\cdot$ Pulmonary embolism . Overview · Diagnostic test

\section{Introduction}

Acute pulmonary embolism (PE) is a common, often undiagnosed and potentially fatal disease. Mortality is between $10 \%$ and $30 \%$ if untreated, but can be reduced to $2-8 \%$ with treatment [1]. Various clinical symptoms and findings together with knowledge of predisposing factors may strengthen or weaken the clinical suspicion. PE can be ruled out if the clinical suspicion is low and the D-dimer test is negative. However, in those patients in whom the clinical suspicion is moderate or high and/or the $\mathrm{D}$-dimer test is positive, a conclusive imaging test such as a ventilation/perfusion $(\mathrm{V} / \mathrm{P})$ study or pulmonary CT angiography (CTPA) is needed to confirm or exclude the diagnosis of PE. There is increasing evidence of the improved accuracy of single photon emission CT (SPECT) and SPECT combined with low-dose CT (SPECT/CT) compared to planar V/P scintigraphy, and likewise of multidetector CTPA compared to single-detector CTPA. There is, however, less evidence concerning comparisons between SPECT and SPECT/CT and CTPA.

In this article, the clinical value and protocols of lung V/P studies with SPECT, SPECT/CT and CTPA are discussed.

\section{Imaging agents}

Ventilation

For ventilation studies with SPECT several kinds of agents can be used: the inert gas ${ }^{81 \mathrm{~m}} \mathrm{Kr}$, ${ }^{99 \mathrm{~m}} \mathrm{Tc}$-based agents such as the carbon suspension Technegas, or radioaerosols, e.g. ${ }^{99 \mathrm{~m}}$ Tc-DTPA. 
${ }^{81 \mathrm{~m}} \mathrm{Kr}$ is delivered from a ${ }^{81} \mathrm{Rb} /{ }^{81 \mathrm{~m}} \mathrm{Kr}$ generator. The short half-life of $13 \mathrm{~s}$ implies that ${ }^{81 \mathrm{~m}} \mathrm{Kr}$ is inhaled continuously during imaging and that it disappears from the alveolar regions by decay much faster than by expiration. Hence, at normal breathing frequency the regional ${ }^{81 \mathrm{~m}} \mathrm{Kr}$ concentration reflects regional ventilation. Due to the higher gamma energy (190.6 keV) of ${ }^{81 \mathrm{~m}} \mathrm{Kr}$ compared to ${ }^{99 \mathrm{~m}} \mathrm{Tc}(140 \mathrm{keV})$, a V/P scan can be acquired simultaneously. The effective radiation dose of ${ }^{81 \mathrm{~m}} \mathrm{Kr}$ is very low $(27 \mathrm{nSv} / \mathrm{MBq},<0.2 \mathrm{mSv}$ for 6 , $000 \mathrm{MBq}$ ). The use of ${ }^{81 \mathrm{~m}} \mathrm{Kr}$ is mainly limited by the distance to a cyclotron since a cyclotron-produced generator only lasts 1 day $\left(T_{1 / 2}\right.$ of $4.6 \mathrm{~h}$ for $\left.{ }^{81} \mathrm{Rb}\right) .{ }^{81 \mathrm{~m}} \mathrm{Kr}$ is very cost-effective if the number of patient examinations is high in a centre, whereas cost is a limitation if only a few patients are examined per generator. ${ }^{133} \mathrm{Xe}$ is rarely used in SPECT due to its low availability and energy.

The ${ }^{99 \mathrm{~m}}$ Tc-labelled Technegas (Cyclomedica Australia, Australia) consists of small ${ }^{99 \mathrm{~m}} \mathrm{Tc}$-graphite particles $(5-200 \mathrm{~nm})$ that are generated by burning solid graphite particles and ${ }^{99 \mathrm{~m}} \mathrm{Tc}$-pertechnetate in argon gas at a high temperature. The particles grow by aggregation and should therefore be administered within $3 \mathrm{~min}$ (no more than $10 \mathrm{~min}$ ). The patient takes one to three deep breaths before imaging and most particles are deposited by diffusion in the alveolar regions, with a biological residence $T_{1 / 2}$ of $135 \mathrm{~h}$. As a result, the time to start imaging, and the duration of image acquisition, are not time-critical [1]. However, due to sedimentation, hotspots may occur in the larger airways and radioactivity may travel up through the airways by mucociliary and cough clearance during the SPECT. The effective radiation dose $(15 \mu \mathrm{Sv} / \mathrm{MBq}, 0.5-0.8 \mathrm{mSv}$ for $30-50 \mathrm{MBq})$ is higher than for ${ }^{81 \mathrm{~m}} \mathrm{Kr}$ and radioaerosols, but lower than for the perfusion agent [1].

Radioaerosols are generated by a nebulizer, preferably creating particles of $1-3 \mu \mathrm{m}$ (mass median aerodynamic diameter). If inhaled slowly they will deposit by sedimentation in the small airways and alveolar regions. The timing and length of imaging examination may be important since retention in the lung depends on the type of ventilation agent used. ${ }^{99 \mathrm{~m}} \mathrm{Tc}-\mathrm{DTPA}$ will clear through the alveolar capillary membrane (through tight-junctions) especially in the dependent lung zones with a $T_{1 / 2}$ of about $60 \mathrm{~min}$ in non-smokers, but two to five times faster in current smokers. Rapid clearance is reflected by the early depiction of radioactivity in the kidneys. Correction for clearance of ${ }^{99 \mathrm{~m}} \mathrm{Tc}$-DTPA during the time span of SPECT has been suggested and applied by some authors [2]. However, correction is not necessary for ${ }^{99 \mathrm{~m}} \mathrm{Tc}$-colloids (e.g. albumen, sulphur) since they have longer residence times in the lungs. Hot-spots in the larger airways occur more often with radioaerosols than with Technegas. The effective radiation dose from radioaerosols is low: $7 \mu \mathrm{Sv} / \mathrm{MBq}, 0.21 \mathrm{mSv}$ for $30 \mathrm{MBq}$ for ${ }^{99 \mathrm{~m}} \mathrm{Tc}-\mathrm{DTPA}$; $4.7 \mu \mathrm{Sv} / \mathrm{MBq}, 0.15 \mathrm{mSv}$ for $30 \mathrm{MBq}$ for ${ }^{99 \mathrm{~m}} \mathrm{Tc}$-albumin colloid (Venticoll) [1]. In centres with only a few V/P studies, radioaerosols are the most costeffective ventilation agents.

\section{Perfusion}

${ }^{99 \mathrm{~m}} \mathrm{Tc}$-labelled macroaggregated human albumin $\left({ }^{99 \mathrm{~m}} \mathrm{Tc}-\right.$ MAA) is injected slowly while the patient breathes slowly and deeply to ensure a uniform perfusion distribution. The administered dose is between 100 and $220 \mathrm{MBq}$ (effective dose $11 \mu \mathrm{Sv} / \mathrm{MBq}, 1.1-2.4 \mathrm{mSv}$ for $100-220 \mathrm{MBq}$ ) and depends mainly on the choice of ventilation agent used [1,3]. A larger dose is required if perfusion imaging is preceded by ${ }^{99 \mathrm{~m}}$ Tc-based ventilation imaging to overcome "background" radiation from the ventilation agent. Indeed, some centres use $300 \mathrm{MBq}^{99 \mathrm{~m}} \mathrm{Tc}-\mathrm{MAA}$ [4] to obtain a required $\mathrm{P} / \mathrm{V}$ ratio of $\geq 4$ when using the ventilation agent Technegas, whereas a lower ${ }^{99 \mathrm{~m}} \mathrm{Tc}$-MAA dose can be used with ${ }^{81 \mathrm{~m}} \mathrm{Kr}$ ventilation, or if the perfusion study is not preceded by a ventilation study [1]. About 400,000 MAA particles are injected, except in patients with pulmonary hypertension or a right-to-left cardiac shunt, and in children, in whom fewer particles are used [5].

\section{Scanning protocols}

\section{V/P SPECT}

The ventilation and perfusion agents for SPECT should be administered with the patient lying supine to reduce any gravitational influence upon distribution. If a ${ }^{99 \mathrm{~m}} \mathrm{Tc}$-based ventilation agent is used, sequential imaging is performed; usually ventilation first, then perfusion. To ensure alignment of the ventilation and perfusion imaging, the patient must not move between the two acquisitions. If ${ }^{81 \mathrm{~m}} \mathrm{Kr}$ is used, V/P imaging can be performed simultaneously (applying a photopeak of $190 \mathrm{keV}$ for ${ }^{81 \mathrm{~m}} \mathrm{Kr}$ and $140 \mathrm{keV}$ for ${ }^{99 \mathrm{~m}} \mathrm{Tc}-$ MAA) ensuring optimal alignment and minimizing acquisition time; alternatively, ${ }^{81 \mathrm{~m}} \mathrm{Kr}$ and ${ }^{99 \mathrm{~m}} \mathrm{Tc}-\mathrm{MAA}$ can be administered and imaged sequentially $[1,5]$.

Below are examples of different V/P SPECT scanning protocols (Table 1):

- A sequential V/P SPECT protocol using ${ }^{99 \mathrm{~m}} \mathrm{Tc}-\mathrm{DTPA}$ radioaerosol $/{ }^{99 \mathrm{~m}} \mathrm{Tc}-\mathrm{MAA}$ [2]. First, the delivered dose from $30 \mathrm{MBq}{ }^{99 \mathrm{~m}} \mathrm{Tc}-\mathrm{DTPA}$ is inhaled into the lungs, and then the V SPECT is acquired. Immediately afterwards, $100 \mathrm{MBq}{ }^{99 m}$ Tc-MAA is given intravenously, and then the P SPECT is acquired. Both SPECT datasets are obtained with 64 projections per head over $180^{\circ}, 10 \mathrm{~s}$ for ventilation and $5 \mathrm{~s}$ for perfusion, using low-energy general-purpose (LEGP) collimators and a $64 \times 64$ matrix. The protocol including both SPECT scans takes $20 \mathrm{~min}$. Ordered-subsets expectation maximization (OSEM) 
reconstruction is performed (two iterations/eight subsets). Since $10-40 \%$ of the ${ }^{99 \mathrm{~m}} \mathrm{Tc}-\mathrm{DTPA}$ is cleared from the lungs during the V SPECT acquisition, this is corrected for. In addition, P SPECT is corrected for "background" radioactivity remaining from the ${ }^{99 \mathrm{~m}} \mathrm{Tc}$-ventilation agent.

- A sequential V/P SPECT protocol using ${ }^{99 \mathrm{~m}} \mathrm{Tc}$ Technegas ${ }^{99 \mathrm{~m}}$ Tc-MAA [3]. First, a dose from $50 \mathrm{MBq}$ ${ }^{99 \mathrm{~m}} \mathrm{Tc}$-Technegas is inhaled into the lungs, and then the $\mathrm{V}$ SPECT is acquired. Immediately afterwards, $220 \mathrm{MBq}$ ${ }^{99 \mathrm{~m}} \mathrm{Tc}-\mathrm{MAA}$ is given intravenously, and then the P SPECT is acquired. Both SPECT datasets are obtained with 60 projections per head over $180^{\circ}, 12 \mathrm{~s}$ for ventilation and $8 \mathrm{~s}$ for perfusion, using low-energy high-resolution (LEHR) collimators and a $128 \times 128$ matrix. The protocol including both SPECT scans takes $25-30 \mathrm{~min}$. OSEM reconstruction is used (eight iterations/four subsets).

- A simultaneous V/P SPECT protocol using ${ }^{81 \mathrm{~m}} \mathrm{Kr} /{ }^{99 \mathrm{~m}} \mathrm{Tc}$ MAA [6]. First, $150 \mathrm{MBq}{ }^{99 \mathrm{~m}} \mathrm{Tc}-\mathrm{MAA}$ is given intravenously, then the V/P SPECT scan is performed, during which ${ }^{81 \mathrm{~m}} \mathrm{Kr}$ is inhaled. Dual energy SPECT is acquired as 36 projections per head of $20 \mathrm{~s}$ each over $180^{\circ}$, using LEGP (or medium-energy general-purpose) collimators and a $128 \times 128$ matrix. The protocol takes $13 \mathrm{~min}$. Iterative reconstruction is performed (three iterations/16 subsets; Astonish, Philips) and images both with attenuation correction (based on a simultaneously obtained lowdose CT scan) and without attenuation correction are reviewed. Our present routine protocol is shortened to less than $10 \mathrm{~min}$ by using $12 \mathrm{~s}$ instead of $20 \mathrm{~s}$ per projection.

\section{SPECT/CT}

The introduction of hybrid SPECT/CT tomography has enabled simultaneous acquisition of V/P SPECT and CT scans of the lungs [9]. CT can be performed as a low-dose CT scan of the lungs and/or as CTPA $[6,9]$. In our centre, the standard routine test for evaluation of PE is combined ${ }^{81 \mathrm{~m}} \mathrm{Kr} /{ }^{99 \mathrm{~m}} \mathrm{Tc}$ MAA (V/P) SPECT/CT (16 slice Precedence; Philips); the CT is performed as a low-dose CT scan without contrast enhancement during free breathing $(140 \mathrm{kVp}, 20 \mathrm{mAs} / \mathrm{slice}$, collimator $16 \times 1.5 \mathrm{~mm}$, rotation time $0.5 \mathrm{~s}$, pitch 0.813 , matrix $512 \times 512)$ immediately before the V/P SPECT scan. The total time required for the combined study is $<15 \mathrm{~min}$. The effective radiation dose from the low-dose CT scan is about $1 \mathrm{mSv}$ [6]. A similar dose of $0.9 \mathrm{mSv}$ from a CT scan $(110 \mathrm{kVp}, 20 \mathrm{mAs} / \mathrm{slice})$ has been reported from a V/P SPECT/CT study from Perth, Australia [10].

In a prospective study comparing simultaneously performed V/P SPECT, V/P SPECT/CT and CTPA [6], we included CTPA ( $80 \mathrm{ml}$ iodine contrast agent, $350 \mathrm{mgI} / \mathrm{ml}$ ) using the bolus tracking technique during a deep inspiration breathhold $(120 \mathrm{kVp}, 230 \mathrm{mAs} / \mathrm{slice}$, collimator $16 \times 0.75 \mathrm{~mm}$, rotation time $0.5 \mathrm{~s}$, pitch $0.94,512 \times 512$ matrix). In our daily 
routine, we occasionally combine V/P SPECT/CT and CTPA in critically ill patients, and in such cases, we start with CTPA. If negative for central PE, we proceed with V/P SPECT/CT to detect any peripheral PE. The combined V/P SPECT/CT and CTPA can be performed in $<20 \mathrm{~min}$.

\section{Pulmonary multidetector CT angiography}

Over the past two decades, CT has undergone rapid development. From single-detector CT to an increasing number of rows of detectors (multidetector CT, MDCT), with an increase in volume coverage in one rotation time and a decrease in the time necessary to acquire the entire chest, all of which have made it possible to image the pulmonary arteries of the lungs in one breath-hold [11]. In the latest generation of CT systems several benefits have been achieved, such as: significant decrease in the volume of iodine contrast agent injected, faster scan time $(<1 \mathrm{~s})$ and lower radiation dose. With the introduction of iterative reconstruction algorithms and faster scan times, it is now possible to lower the peak kilovoltage from 120 to $80 \mathrm{kVp}$ and, with the use of dose modulation software, to decrease the radiation dose in selected systems by $>70 \%$ without compromising the signal-to-noise ratio [12]. An effective dose for CTPA of $\leq 1 \mathrm{mSv}$ is now achievable with the state-of-the-art systems available [13].

The development of faster scan times has further resulted in a decreased number of inconclusive scans from approximately $10 \%$ to $2 \%$. These are most often due to patient movement or poor bolus enhancement [14-16]. Additionally, continuous improvement of the contrast agent injection protocols has to be achieved due to the shorter scan times. The paramount challenge is in timing the intravenous injection of the contrast agent bolus and accomplishing high contrast enhancement in the pulmonary vessels and at the same time lowering the volume of contrast agent administered to reduce the adverse effects of iodine (Fig. 1). Consequently, the volume of iodine contrast agent required can be reduced to $60-70 \mathrm{ml}$ $(300-400 \mathrm{mg} \mathrm{I} / \mathrm{ml})$ with the fast high-end systems compared to $150 \mathrm{ml}$ with older systems ( $2-16$ detectors $)$. Nonetheless, impairment of kidney function is still an important issue with iodine contrast agents.

The latest improvement in $\mathrm{CT}$ is the development of dual energy CT systems. A dual energy CT system with two independent tube detector systems (e.g. $140 \mathrm{kVp}$ and $80 \mathrm{kVp}$ ) makes material differentiation possible. Iodine distribution can be visualized in the parenchyma of the lung and can reveal perfusion changes and has the potential to reveal subsegmental clots. In addition, stable xenon gas can be used in dual energy CT systems for CT ventilation imaging, mimicking the ventilation scan obtained in lung scintigraphy [17]. Hence, it is now feasible to accomplish functional information (perfusion/ventilation) combined with morphological imaging in the same lung CT scan $[18,19]$. However, further studies have to be done before this can be applied in routine clinical practice.

\section{Clinical value}

\section{V/P SPECT}

V/P SPECT overcomes the superimposition of lung areas with normal perfusion onto areas with perfusion defects, which may mask PE. It also allows recognition of perfusion and ventilation defects typical of other diseases. Many studies demonstrate enhanced sensitivity and specificity, as well as a reduction in the nondiagnostic rate of V/P SPECT compared with planar V/P scintigraphy [4, 20-23] (Table 2). In clinical head-to-head comparisons of V/P SPECT and planar scintigraphy, more and smaller defects were seen on SPECT and higher sensitivity, specificity and accuracy were also observed: the median differences between SPECT and planar V/P scans in sensitivity, specificity and accuracy were $21 \%$ (range 15 to $36 \%$ ), $6 \%$ (range -7 to $15 \%$ ) and $7 \%$ (range -2 to $20 \%$ ), respectively [20-22]. A substantial proportion of V/P planar studies are usually indeterminate (up to $73 \%$ in PIOPED1), but the addition of V/P SPECT renders the test diagnostic in most patients [4]. V/P SPECT is diagnostic in $\geq 95 \%$ of
Fig. 1 Bilateral central pulmonary emboli on the $\mathrm{CT}$ scan. Axial and coronal CT images with intravenous contrast agent administration show large contrast defects in the central pulmonary arteries (arrows). Note the lack of contrast enhancement in the small subsegmental pulmonary arteries
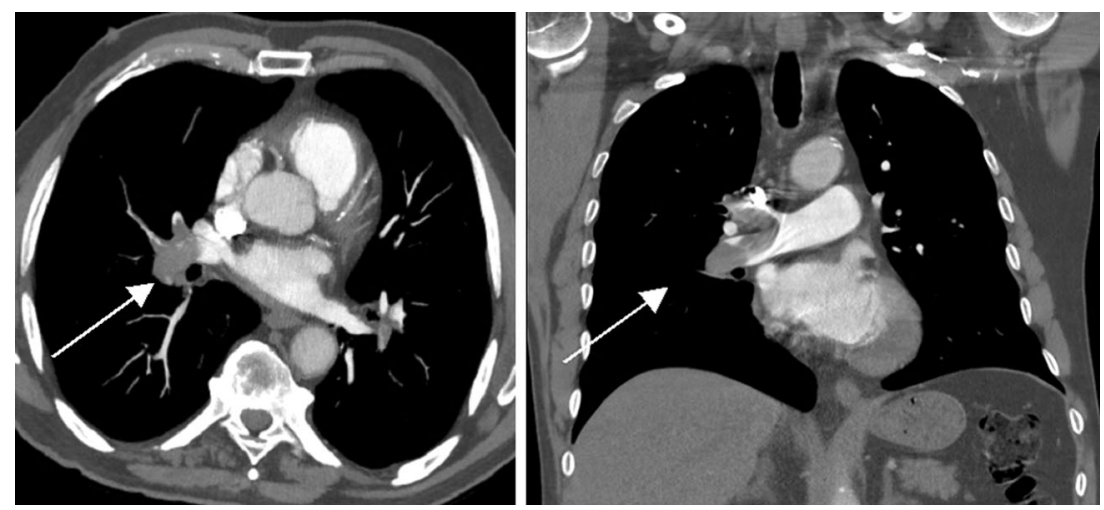


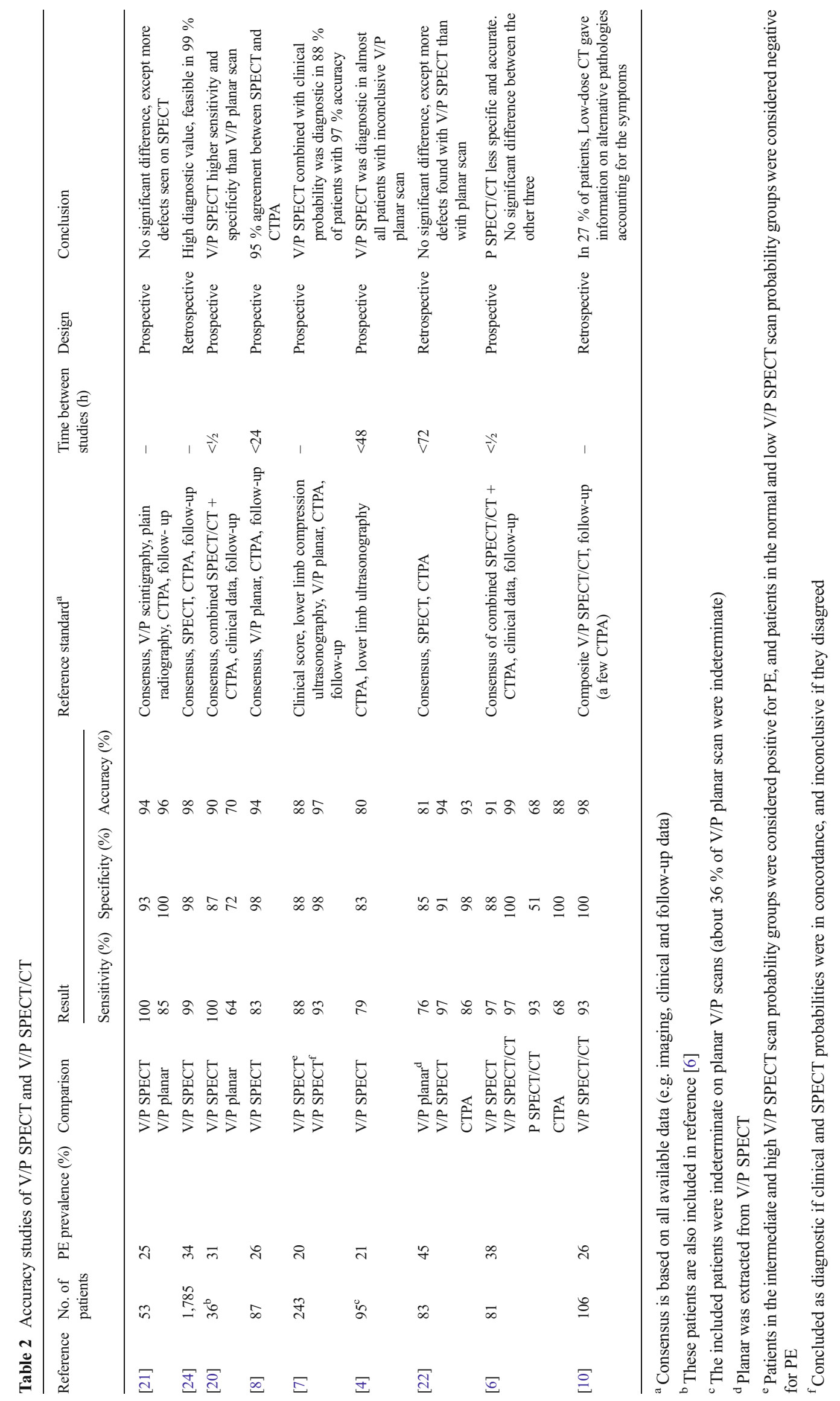


patients and is feasible in $97-99 \%$ of patients referred for lung scintigraphy [6, 24-26]. V/P planar scintigraphy remains an alternative in the few patients in whom SPECT cannot be performed due to for example obesity or discomfort in the supine position [24].

Some patterns on V/P SPECT may suggest alternative diagnoses such as COPD, pneumonia, and left heart diseases $[24,27,28]$ but even if these diseases are present it is possible to diagnose PE with V/P SPECT [1]. A recent study showed that if the reader has extensive experience with PISA-PED readings of perfusion scintigraphy, it is also possible to obtain high diagnostic accuracy ( $\geq 90 \%$ ) for PE from perfusion SPECT only without ventilation information [29].

\section{Interpretation criteria of V/P SPECT}

Iterative reconstruction is recommended (for example using OSEM, with e.g. eight subsets and two iterations) [1]. Perfusion and ventilation images in axial, coronal and sagittal projections and rotating 3-D images are read simultaneously. As an option, V/P ratio images can also be displayed [1] as well as low-dose CT images, if available [6] (Fig. 2).

Different diagnostic cut-off criteria have been used in the published V/P SPECT studies. Le Duc-Pennec et al. applied the revised PIOPED criteria as proposed by Gottschalk et al. for planar V/P scan interpretation $[7,30]$. In a reanalysis of 249 V/P SPECT datasets assessing various combinations of mismatched defect numbers and sizes from segmental to subsegmental, the same authors found that a diagnostic cutoff of one segmental or two subsegmental mismatches was best for confirming or excluding acute PE with a sensitivity of $92 \%$ and specificity of $91 \%$ [31]. Combining pretest clinical probability with SPECT for a negative V/P SPECT result gave post-test probabilities of PE of $1 \%, 4 \%$ and $12 \%$ for low, intermediate and high clinical probability, respectively. For a positive V/P SPECT result, post-test probabilities of PE were $53 \%, 81 \%$ and $94 \%$ for low, intermediate and high clinical probability, respectively (Table 2 ).

Leblanc et al. applied the diagnostic criteria for PE of "any clear-cut vascular mismatch, regardless of size", which had a negative predictive value of $98 \%$ at 3 months [26]. An almost similar diagnostic cut-off of wedge-shaped pleural-based V/P mismatch of $\geq 0.5$ segment has been used by others $[6,8]$ and has recently been shown to be the best value in a V/P planar study [32]. A third approach was a cut-off of the presence of $>50 \%$ perfusion mismatch in an anatomical segment or two regions of perfusion mismatch, regardless of size [10].

The European (EANM) guidelines from 2009 recommend that "PE" is reported if there is V/P mismatch of one or more segments or two subsegments that conform to the pulmonary vascular anatomy. "No PE" is reported if there is a normal perfusion pattern conforming to the anatomic boundaries of the lung, matched or reversed mismatch V/P defects (any size, shape, number), or a mismatch that does not have a subsegmental, segmental or lobar pattern. "Non-diagnostic for PE" is reported if there are multiple V/P abnormalities not typical of specific disease [1].

\section{Clinical validity of PE-negative V/P SPECT studies and clinical outcome}

The negative predictive value of V/P SPECT for PE is $\geq 97 \%$ [6, 24-26]. The long-term outcome of withholding antithrombotic treatment after a negative SPECT scan has been studied $[25,26]$ in 1,159 and 405 patients and only $0.5-1.5 \%$ of the scans were false-negative $[24,26]$, similar to reported values for V/P planar studies [33-35].

\section{Follow-up with V/P SPECT}

In the same manner as the use of $\mathrm{V} / \mathrm{P}$ planar lung scintigraphy for follow-up after large PE, SPECT should be ideal for follow-up studies due to its higher resolution and accuracy. A prospective study of 23 patients with PE showed the feasibility of follow-up with V/P SPECT for evaluating treatment effectiveness and identifying patients who develop chronic PE. Resolution of perfusion defects after PE occurred within the first 3 months of treatment [36].

\section{V/P SPECT/CT}

The accuracy of V/P SPECT combined with low-dose CT has been assessed in two studies (Table 2). In a prospective study, simultaneously obtained V/P SPECT, V/P SPECT/CT and CTPA were compared and PE was diagnosed in $38 \%$ using head-to-head comparisons and 6 months follow-up [6]. V/P SPECT and V/P SPECT/CT had the highest sensitivity (97\%), whereas V/P SPECT/CT and CTPA had the highest specificity $(100 \%)$, and V/P SPECT/CT had the highest accuracy (99\%). Differences were not significantly different, however, due to the relatively small number of patients. A test that included V/P SPECT gave the highest sensitivity, while a test that included CT, either CTPA or low-dose CT integrated with V/P SPECT, gave the best specificity and was always either positive or negative for PE (100\% diagnostic rate) and could often give alternative diagnoses. Adding low-dose CT to V/P SPECT increased specificity (from $88 \%$ to $100 \%$ ) by reducing false-positive interpretations (from $18 \%$ to $0 \%$ ), without affecting sensitivity (still $97 \%$ ). Low-dose CT improved confidence in reading and reduced the inconclusive assessments from $5 \%$ to $0 \%$. The improvement in specificity was mainly due to low-dose $\mathrm{CT}$ findings providing alternative explanations for subtle perfusion defects such as small interlobar fissures, localized hyperinflated areas, paraseptal emphysema, pleural fluid, atelectasis and pneumonic infiltration, which would otherwise be read as PE on SPECT (Fig. 3). 
Fig. 2 A pulmonary embolus is seen on the V/P SPECT/CT images. A wedge-shaped pleuralbased large mismatched perfusion defect is seen anteriorly in the left lung (yellow arrows) on the axial (upper row), coronal (middle row) and sagittal (lower row) projections. No other explanations for the perfusion defect can be seen on the normal ventilation scan and low-dose CT images
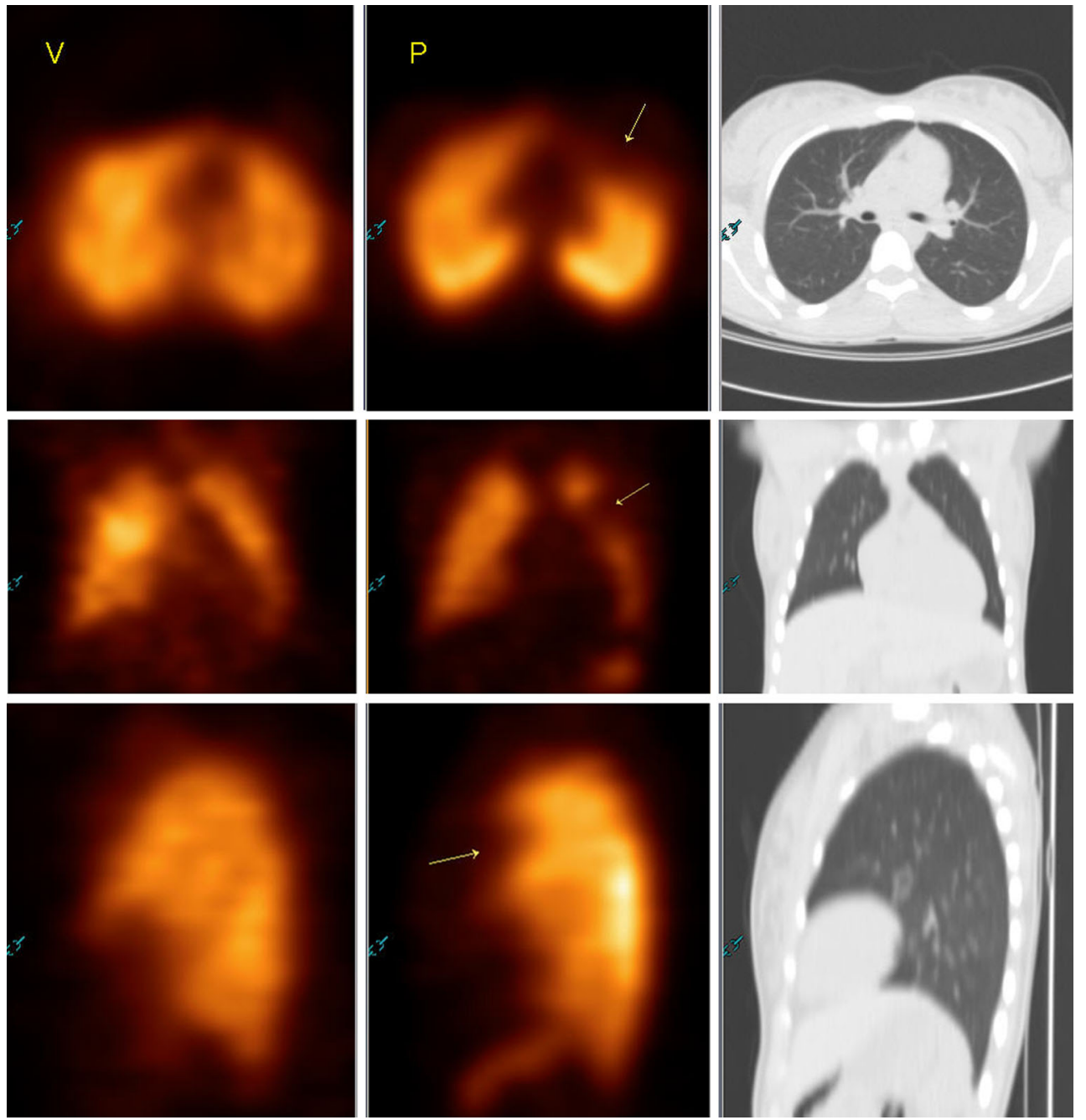

However, low-dose CT combined with perfusion SPECT (without ventilation SPECT) was suboptimal due to a low specificity (51\%) and positive predictive value (57\%) and a high nondiagnostic rate $(17 \%)$, while sensitivity and negative predictive value remained over $90 \%$. Hence, ventilation SPECT is needed in addition to perfusion SPECT when using low-dose CT.

Ling et al. performed V/P SPECT/CT in 106 patients using a composite reference standard for PE including a 6-month follow-up, but CTPA was only used in a few patients with normal V/P scans but high clinical suspicion [10]. PE was diagnosed in $26 \%$ of patients, including $2 \%$ with a falsenegative V/P SPECT/CT result. The negative predictive value of V/P SPECT for PE was $97 \%$. Abnormalities detected by low-dose CT were correlated with the V/P SPECT findings to suggest alternative diagnoses in those negative for PE. Lowdose CT was abnormal in $41 \%$ of patients, and in $27 \%$ of all patients low-dose CT provided information on alternative
Fig. 3 Very small mismatched perfusion defects (yellow arrows) are seen peripherally in both lungs, but the CT image shows widespread pleural based interstitial ground-glass abnormalities (blue arrows) compatible with inflammation, but not with PE
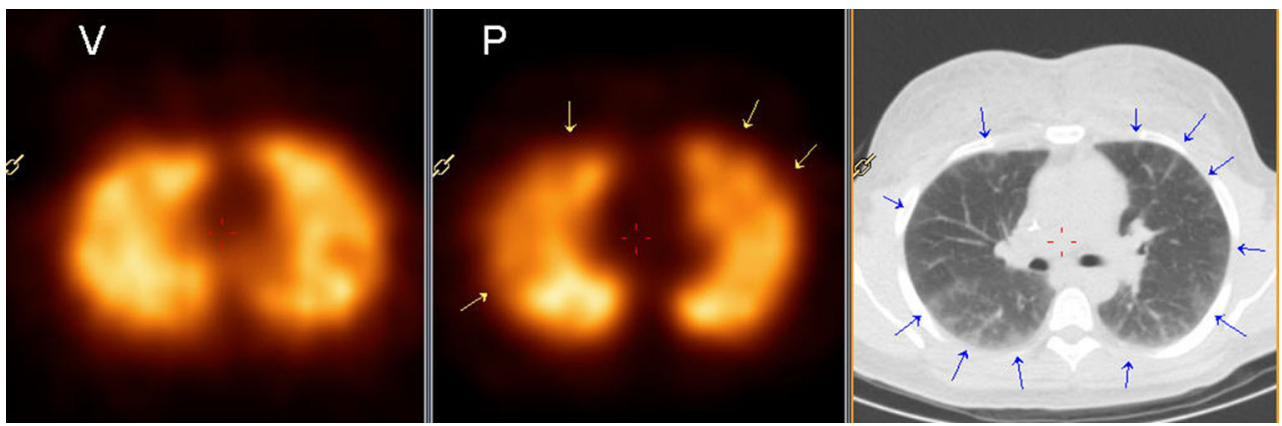
pathologies that accounted for the symptoms such as pneumonia, pulmonary oedema and ARDS, corresponding to the CTPA findings [37]. Performing low-dose CT allows attenuation correction of the SPECT data. The value of attenuation correction of V/P SPECT studies in PE is, however, unresolved [3].

Another potential combination of functional and structural information from V/P SPECT and CT is a fusion of SPECT and CTPA scan acquired on the same or separate systems [9, 38]. Accurate fusion of SPECT and CTPA may be clinically useful in nondiagnostic initial investigations, or where corroborative imaging is sought.

\section{Pulmonary CT angiography}

Several studies have demonstrated that CTPA has higher diagnostic accuracy and specificity than planar V/P scintigraphy $[19,37]$. In a study with 179 patients, Blachere et al. demonstrated that sensitivity, specificity, positive predictive value and negative predictive value of CTPA and planar V/P scans were $94 \%$ versus $80 \%, 93 \%$ versus $73 \%, 95 \%$ versus $82 \%$ and $96 \%$ versus $75 \%$, respectively [19]. Similar results were reported by Grenier and Beigelman [39] and Mayo et al. [40]. The strength of V/P scintigraphy is its high negative predictive value in patients with a low pretest probability and its high positive predictive value in patients with a high pretest probability [30]. Hence, it was part of the composite gold standard for diagnosing PE in the PIOPED2 study, in which CTPA had a sensitivity of $83 \%$ and specificity of $96 \%$ and was inconclusive in $6 \%$ [14].

The number of head-to-head comparisons between V/P SPECT and CTPA is still limited (Table 2) $[6,8,22]$. There is a $95 \%$ concordance between V/P SPECT and CTPA [8], with a higher sensitivity with SPECT, a higher specificity with CTPA, and equally high accuracy with both methods $[6,22]$. The combination of V/P SPECT and low-dose CT may be even more accurate than V/P SPECT or CTPA, but more studies are warranted [6].

V/P SPECT and V/P SPECT/CT are available in many nuclear medicine departments, mostly during routine working hours. They can provide alternative diagnoses, have high interobserver agreement, and can be performed in almost all patients since there are no definitive contraindications $[1,41]$. CTPA is available in most clinical centres round-the-clock. It is capable of providing alternative diagnoses, has faster scan times, has high interobserver agreement and could have a possible role in detecting right ventricular dysfunction. It does have contraindications, however, and cannot be performed in $10-30 \%$ of inpatients $[6,11,14,42]$.

Patients with PE may have multiple scans due to the risk of recurrence or due to comorbidity, and therefore are at risk from cumulative exposure to ionizing radiation. Especially in young women, the radiation dose to the breast tissue is of critical importance. Chest CT scanning results in relatively higher doses to the breast tissue than lung scintigraphy. However, the latest iterative reconstruction technique can lead to a $60-80 \%$ reduction in dose, and at the same time, a $43-80 \%$ improvement in low-contrast detectability and a $70-83 \%$ reduction in image noise, compared with standard (filtered back projection) reconstruction. Therefore, radiation dose to the breast can be reduced $[13,43]$. However, there is no broad consensus on this issue and further research is needed.

\section{Clinical validity of PE-negative CT studies and clinical outcome}

Carrier et al. demonstrated in a systematic review that multiple detector CTPA (2 - 64 detectors) might increase the rate of subsegmental PE diagnosis as compared with single detector CTPA. They also demonstrated that the 3-month thromboembolic risk in patients with suspected PE who were left untreated after a negative CTPA result was similar regardless of whether the patients underwent a single or multiple detector CTPA. Finally, they concluded that the incremental rate of subsegmental PE detected by multiple detector CTPA might not be clinically important [44].

A meta-analysis by Quiroz et al. with pooled results involving 15 studies and 3,500 patients with suspected PE suggested that clinical outcome is not adversely affected if anticoagulant therapy is withheld based on a negative CT scan with a negative predictive value of $99.1 \%$ [45]. These studies were performed with older generation MDCT systems, and it is therefore possible that subsegmental pulmonary emboli remained undetected. Newer high-end CT systems have a higher spatial and temporal resolution and are therefore able to visualize smaller subsegmental emboli. However, consensus regarding the treatment of isolated subsegmental emboli in patients with suspected PE has not been established and whether this will result in improved clinical outcome is debatable [46]. Since subsegmental PE may precede recurrent large PE and may also increase the risk of development of chronic pulmonary hypertension, improvement in the detection of subsegmental PE is worth studying [18].

\section{Conclusion}

Compared with traditional V/P scintigraphy, both V/P SPECT and CTPA have a higher diagnostic value and fewer inconclusive results in the diagnosis of PE. V/P SPECT and CTPA are highly concordant and show similar diagnostic accuracy, with SPECT showing higher sensitivity and CTPA higher specificity. There is possibly a higher clinical value of negative SPECT studies compared to negative CTPA studies, but the clinical importance of finding a small subsegmental PE needs further elucidation. A combination of V/P SPECT and 
CT may be even more accurate than V/P SPECT or CTPA alone. More comparisons are needed, however. Each of these excellent imaging methods has the highest predictive values when there is concordance between the clinical suspicion and the test result, whereas further imaging should be performed if there is discordance.

V/P SPECT or V/P SPECT/CT are available in many nuclear medicine departments, mostly during the day-time. They may provide an alternative diagnosis if PE is refuted, and are feasible in almost all patients, because there are no definitive contraindications. CTPA is usually available roundthe-clock and a rapid PE or alternative diagnosis can be expected. Due to contraindications such as kidney impairment or allergy, CTPA cannot be performed in about $10-30 \%$ of patients. Whereas radiation exposure was previously substantially higher for the patient with CTPA than with V/P SPECT or $\mathrm{V} / \mathrm{P}$ SPECT/CT, a much lower radiation dose is now achievable with new generation CT technology.

\section{Conflicts of interest None.}

Open Access This article is distributed under the terms of the Creative Commons Attribution License which permits any use, distribution, and reproduction in any medium, provided the original author(s) and the source are credited.

\section{References}

1. Bajc M, Neilly JB, Miniati M, Schuemichen C, Meignan M, Jonson B. EANM guidelines for ventilation/perfusion scintigraphy: Part 1 . Pulmonary imaging with ventilation/perfusion single photon emission tomography. Eur J Nucl Med Mol Imaging. 2009;36(8):1356-70.

2. Palmer J, Bitzen U, Jonson B, Bajc M. Comprehensive ventilation/ perfusion SPECT. J Nucl Med. 2001;42(8):1288-94.

3. Roach PJ, Bailey DL, Harris BE. Enhancing lung scintigraphy with single-photon emission computed tomography. Semin Nucl Med. 2008;38(6):441-9.

4. Weinmann P, Moretti J-L, Brauner MW. Usefulness of tomographic versus planar lung scintigraphy in suspected pulmonary embolism in a daily practice. Open Med Imaging J. 2013;2:49-55.

5. Bajc M, Neilly B, Miniati M, Mortensen J, Jonson B. Methodology for ventilation/perfusion SPECT. Semin Nucl Med. 2010;40(6):415-25.

6. Gutte H, Mortensen J, Jensen CV, Johnbeck CB, von der Recke P, Petersen CL, et al. Detection of pulmonary embolism with combined ventilation-perfusion SPECT and low-dose CT: head-to-head comparison with multidetector CT angiography. J Nucl Med. 2009;50(12):1987-92.

7. Le Duc-Pennec A, Le Roux PY, Cornily JC, Jaffrelot M, Delluc A, de Saint-Martin L, et al. Diagnostic accuracy of single-photon emission tomography ventilation/perfusion lung scan in the diagnosis of pulmonary embolism. Chest. 2012;141(2):381-7.

8. Miles S, Rogers KM, Thomas P, Soans B, Attia J, Abel C, et al. A comparison of single-photon emission CT lung scintigraphy and CT pulmonary angiography for the diagnosis of pulmonary embolism. Chest. 2009;136(6):1546-53.

9. Gutte H, Mortensen J, Jensen C, von der Recke P, Kristoffersen US, Kjaer A. Added value of combined simultaneous lung ventilation- perfusion single-photon emission computed tomography/multi-slicecomputed tomography angiography in two patients suspected of having acute pulmonary embolism. Clin Respir J. 2007;1(1):52-5.

10. Ling IT, Naqvi HA, Siew TK, Loh NK, Ryan GF. SPECT ventilation perfusion scanning with the addition of low-dose CT for the investigation of suspected pulmonary embolism. Int Med J. 2012;42(11): 1257-61.

11. Reid JH, Coche EE, Inoue T, Kim EE, Dondi M, Watanabe N, et al. Is the lung scan alive and well? Facts and controversies in defining the role of lung scintigraphy for the diagnosis of pulmonary embolism in the era of MDCT. Eur J Nucl Med Mol Imaging. 2009;36(3):505-21.

12. Li Q, Yu H, Zhang L, Fan L, Liu S. Combining low tube voltage and iterative reconstruction for contrast-enhanced CT imaging of the chest - initial clinical experience. Clin Radiol. 2013;68(5):e249-53.

13. Mehta D, Thompson R, Morton T, Dhanantwari A, Shefer E. Iterative model reconstruction: simultaneously lowered computed tomography radiation dose and improved image quality. Med Phys Int J. 2013;2(1):147-55.

14. Stein PD, Fowler SE, Goodman LR, Gottschalk A, Hales CA, Hull $\mathrm{RD}$, et al. Multidetector computed tomography for acute pulmonary embolism. N Engl J Med. 2006;354(22):2317-27.

15. Jones SE, Wittram $\mathrm{C}$. The indeterminate CT pulmonary angiogram: imaging characteristics and patient clinical outcome. Radiology. 2005;237(1):329-37.

16. Pesavento R, de Conti G, Minotto I, Filippi L, Mongiat M, de Faveri $\mathrm{D}$, et al. The value of 64-detector row computed tomography for the exclusion of pulmonary embolism. Thromb Haemost. 2011;105(5):901-7.

17. Thieme S, Hoegl S, Nikolaou K, Fisahn J, Irlbeck M, Maxien D, et al. Pulmonary ventilation and perfusion imaging with dual-energy CT. Eur Radiol. 2010;20(12):2882-9.

18. Pontana F, Faivre JB, Remy-Jardin M, Flohr T, Schmidt B, Tacelli N, et al. Lung perfusion with dual-energy multidetector-row CT (MDCT): feasibility for the evaluation of acute pulmonary embolism in 117 consecutive patients. Acad Radiol. 2008;15(12):1494-504.

19. Blachere H, Latrabe V, Montaudon M, Valli N, Couffinhal T, Raherisson C, et al. Pulmonary embolism revealed on helical CT angiography: comparison with ventilation-perfusion radionuclide lung scanning. AJR Am J Roentgenol. 2000;174(4):1041-7.

20. Gutte H, Mortensen J, Jensen CV, von der Recke P, Petersen CL, Kristoffersen US, et al. Comparison of V/Q SPECT and planar V/Q lung scintigraphy in diagnosing acute pulmonary embolism. Nucl Med Commun. 2010;31(1):82-6.

21. Bajc M, Olsson CG, Olsson B, Palmer J, Jonson B. Diagnostic evaluation of planar and tomographic ventilation/perfusion lung images in patients with suspected pulmonary emboli. Clin Physiol Funct Imaging. 2004;24(5):249-56.

22. Reinartz P, Wildberger JE, Schaefer W, Nowak B, Mahnken AH, Buell U. Tomographic imaging in the diagnosis of pulmonary embolism: a comparison between V/Q lung scintigraphy in SPECT technique and multislice spiral CT. J Nucl Med. 2004;45(9):1501-8.

23. Bajc M, Bitzen U, Olsson B, Perez de Sa V, Palmer J, Jonson B. Lung ventilation/perfusion SPECT in the artificially embolized pig. J Nucl Med. 2002;43(5):640-7.

24. Bajc M, Olsson B, Palmer J, Jonson B. Ventilation/perfusion SPECT for diagnostics of pulmonary embolism in clinical practice. J Intern Med. 2008;264(4):379-87.

25. Corbus HF, Seitz JP, Larson RK, Stobbe DE, Wooten W, Sayre JW, et al. Diagnostic usefulness of lung SPET in pulmonary thromboembolism: an outcome study. Nucl Med Commun. 1997;18(10): 897-906.

26. Leblanc M, Leveillee F, Turcotte E. Prospective evaluation of the negative predictive value of V/Q SPECT using 99mTc-Technegas. Nucl Med Commun. 2007;28(8):667-72.

27. Jogi J, Palmer J, Jonson B, Bajc M. Heart failure diagnostics based on ventilation/perfusion single photon emission computed tomography 
pattern and quantitative perfusion gradients. Nucl Med Commun. 2008;29(8):666-73.

28. Bajc M, Jonson B. Ventilation/perfusion SPECT for diagnosis of pulmonary embolism and other diseases. Int J Mol Imaging. 2011;2011:682949.

29. Bajc M, Miniati M, Jogi J, Stein PD. Perfusion SPECT in patients with suspected pulmonary embolism. Eur J Nucl Med Mol Imaging. 2013;40(9):1432-7.

30. Gottschalk A, Sostman HD, Coleman RE, Juni JE, Thrall J, McKusick KA, et al. Ventilation-perfusion scintigraphy in the PIOPED study. Part II. Evaluation of the scintigraphic criteria and interpretations. J Nucl Med. 1993;34(7):1119-26.

31. Le Roux PY, Robin P, Delluc A, Abgral R, Le Duc-Pennec A, Nowak $\mathrm{E}$, et al. V/Q SPECT interpretation for pulmonary embolism diagnosis: which criteria to use? J Nucl Med. 2013;54(7):1077-81.

32. Howarth DM, Booker JA, Voutnis DD. Diagnosis of pulmonary embolus using ventilation/perfusion lung scintigraphy: more than 0.5 segment of ventilation/perfusion mismatch is sufficient. Int Med J. 2006;36(5):281-8.

33. Hull RD, Raskob GE, Coates G, Panju AA. Clinical validity of a normal perfusion lung scan in patients with suspected pulmonary embolism. Chest. 1990;97(1):23-6.

34. van Beek EJ, Kuyer PM, Schenk BE, Brandjes DP, ten Cate JW, Buller HR. A normal perfusion lung scan in patients with clinically suspected pulmonary embolism. Frequency and clinical validity. Chest. 1995;108(1):170-3.

35. Anderson DR, Kahn SR, Rodger MA, Kovacs MJ, Morris T, Hirsch A, et al. Computed tomographic pulmonary angiography vs ventilationperfusion lung scanning in patients with suspected pulmonary embolism: a randomized controlled trial. JAMA. 2007;298(23):2743-53.

36. Begic A, Jogi J, Hadziredzepovic A, Kucukalic-Selimovic E, Begovic-Hadzimuratovic S, Bajc M. Tomographic ventilation/ perfusion lung scintigraphy in the monitoring of the effect of treatment in pulmonary embolism: serial follow-up over a 6-month period. Nucl Med Commun. 2011;32(6):508-14.

37. Coche E, Verschuren F, Keyeux A, Goffette P, Goncette L, Hainaut $\mathrm{P}$, et al. Diagnosis of acute pulmonary embolism in outpatients: comparison of thin-collimation multi-detector row spiral CT and planar ventilation-perfusion scintigraphy. Radiology. 2003;229(3):757-65.

38. Harris B, Bailey D, Roach P, Bailey E, King G. Fusion imaging of computed tomographic pulmonary angiography and SPECT ventilation/perfusion scintigraphy: initial experience and potential benefit. Eur J Nucl Med Mol Imaging. 2007;34(1):135-42.

39. Grenier PA, Beigelman C. Ventilation-perfusion scintigraphy in the PIOPED study. Part II. Evaluation of the scintigraphic criteria and interpretations. J Nucl Med. 1993;34(7):1119-26.

40. Mayo JR, Remy-Jardin M, Muller NL, Remy J, Worsley DF, Hossein-Foucher C, et al. Pulmonary embolism: prospective comparison of spiral CT with ventilation-perfusion scintigraphy. Radiology. 1997;205(2):447-52.

41. Bajc M, Neilly JB, Miniati M, Schuemichen C, Meignan M, Jonson B. EANM guidelines for ventilation/perfusion scintigraphy: Part 2. Algorithms and clinical considerations for diagnosis of pulmonary emboli with V/P(SPECT) and MDCT. Eur J Nucl Med Mol Imaging. 2009;36(9):1528-38.

42. Gutte H, Mortensen J, Jensen CV, von der Recke P, Petersen CL, Kristoffersen US, et al. ANP, BNP and D-dimer predict right ventricular dysfunction in patients with acute pulmonary embolism. Clin Physiol Funct Imaging. 2010;30(6):466-72.

43. Mettler Jr FA, Huda W, Yoshizumi TT, Mahesh M. Effective doses in radiology and diagnostic nuclear medicine: a catalog. Radiology. 2008;248(1):254-63.

44. Carrier M, Righini M, Wells PS, Perrier A, Anderson DR, Rodger MA, et al. Subsegmental pulmonary embolism diagnosed by computed tomography: incidence and clinical implications. A systematic review and meta-analysis of the management outcome studies. J Thromb Haemost. 2010;8(8):1716-22.

45. Quiroz R, Kucher N, Zou KH, Kipfmueller F, Costello P, Goldhaber SZ, et al. Clinical validity of a negative computed tomography scan in patients with suspected pulmonary embolism. A systematic review. JAMA. 2005;293(16):2012-7.

46. Stein PD, Goodman LR, Hull RD, Dalen JE, Matta F. Diagnosis and management of isolated subsegmental pulmonary embolism: review and assessment of the options. Clin Appl Thromb Hemost. 2012;18(1):20-6. 